\title{
Análisis bibliométrico de intervenciones basadas en terapia de tercera generación para niños y adolescentes en Iberoamérica y Europa*
}

\author{
Bibliometric analysis of interventions based on third \\ generation therapies in children and adolescents in \\ Iberoamerica and Europe
}

\section{Sonia Zambrano Hernández** \\ Lizeth Tatiana Camargo Hernández \\ Johana Caterine Jerez-Castiblanco Universidad Católica de Colombia \\ Geraldine Lizeth Gómez Padilla Fuerza Aérea Colombiana \\ Laura Fernanda Perea-Gil Pontificia Universidad Javeriana}

Recibido: 6 de junio de 2017 Revisado: 1 de agosto de 2017 Aceptado: 2 de septiembre de 2017

\section{Resumen}

El presente estudio es un análisis bibliométrico de las publicaciones en intervenciones basadas en las terapias de tercera generación en niños y adolescentes durante los años 2011-2016. Para esto se realizó un estudio descriptivo dentro del cual se analizaron 60 artículos científicos de diversas bases de datos como Proquest, Redalyc, Sage, ScienceDirect y Springer Science, Dialnet, Ebsco Host. Se evidenció que entre los años 2012 y 2014 se presentó un incremento en las publicaciones de terapias de tercera generación en Iberoamérica y Europa. La revista International Journal of Behavioral Consultation \& Therapy presenta la mayor cantidad de publicaciones en este ámbito. En relación con el autor que presenta más publicaciones, se referencia a Swart, $\mathrm{J}$ como el principal. La revisión teórica y el estudio cuasi experimental fueron la metodología más reportada y el Mindfulness la terapia más utilizada. En cuanto al país con Mayor número de investigaciones en esta temática es Estados Unidos. Este estudio

Artículo de investigación. http://dx.doi.org/10.15332/s1794-9998.2018.0001.06

Correspondencia: Sonia Zambrano, Profesora, Facultad de Psicología, Universidad Católica de Colombia. Dirección Postal: Avenida Caracas \# 46 - 72, Bogotá, Colombia. Correo electrónico: szambranoh@gmail.com 
permite concluir que las terapias de tercera generación han comenzado a tomar fuerza en varios de los problemas comportamentales y emocionales en niños y adolescentes. Sin embargo, es necesario ampliar las investigaciones en este ámbito dado que en la mayoría de los artículos no se cuantifica el grado de eficacia.

Palabras clave: Análisis bibliométrico, terapia de aceptación y compromiso, niños y jóvenes.

\section{Abstract}

The present study is a bibliometric analysis of publications on interventions based on third generation therapies in children and adolescents during the years 2011-2016. For this, a descriptive study was carried out in which 60 scientific articles from diverse databases were analyzed, such as Proquest, Redalyc, Sage, ScienceDirect and Springer Science, Dialnet, Ebsco Host. It was evidenced that between the years 2012 and 2014 there was an increase in the publications of third generation therapies in Ibero-America and Europe. The International Journal of Behavioral Consultation $\&$ Therapy presents the largest number of publications in this field. In relation to the author who presents the most publications, reference is made to Swart, $\mathrm{J}$ as the principal. The theoretical review and the quasi-experimental study were the most reported methodology and Mindfulness the most used therapy. As for the country with the largest number of investigations in this area is the United States. This study allows us to conclude that third generation therapies have begun to gain strength in several behavioral and emotional problems in children and adolescents. However, it is necessary to expand the research in this field since in most articles the degree of efficacy is not quantified.

Keywords: bibliometric analysis, acceptance and commitment therapy, children and youth.

\section{Introducción}

La tercera generación se da inicio en la década de 1990 no siendo conocida hasta el 2004. (Hayes, 2004). Esta intervención se encuentra fundamentada en una aproximación empírica enfocada en los principios del aprendizaje. "Considera que la tercera ola de terapias cognitivas y conductuales es particularmente sensible al contexto y a las funciones de los fenómenos psicológicos, y no sólo a la forma, enfatizando el uso de estrategias de cambio basadas en la experiencia y en el contexto además de otras más directas y didácticas". (Moreno, 2012)

Las investigaciones relacionadas con las terapias de tercera generación aún son muy recientes. Sin embargo, se han encontrado en estas, resultados que dan cuenta de su eficacia para intervenir en los problemas psicológicos (Moreno, 2012). Aun- que se presenta un número reducido de estudios, han demostrado la eficacia en el abordaje de problemáticas en la salud mental infantil.

Los estudios en las terapias de tercera generación toman aportes principalmente de las terapias conductistas radicales (Hayes, 2004). En este tipo de terapias, surge una nueva forma de comprender los problemas psicológicos que se conoce como una perspectiva contextualista. En la cual, se plantea que los eventos externos que generan malestar surgen en un contexto que se relaciona con eventos internos, $y$, al tratar de suprimir o eliminar estos eventos se propicia el aumento del malestar y se mantiene el problema inicial (Hayes, 2004).

Dentro las terapias de este tipo, se encuentra la terapia de aceptación y compromiso. Esta realiza una crítica al sistema social, que no ve el sufrimiento como parte de la vida, sino como un as- 
pecto indeseado que debe ser suprimido. Desde esta terapia se busca que las personas aprendan a aceptar que muchos de los eventos negativos que suceden les pueden generan malestar emocional y que este proceso hace parte de la vida misma (Ribero \& Agudelo, 2016).

Ya dentro del enfoque de compromiso estaría no permitir que estos eventos definan a la persona. Sino que el individuo pueda observar el aquí y el ahora, y entender que estos pensamientos hacen parte de una interpretación que se da, pero que puede cambiarse su función y contexto. Además de lo anterior, se da orientación a que direccionen su vida, en donde están sus valores individuales (Ribero \& Agudelo, 2016).

Las terapias de tercera generación que mayormente se mencionan son la Terapia de Aceptación y Compromiso (ACT), Mindfulness, definida como la práctica en la que tomamos conciencia de las distintas facetas de nuestra experiencia en el momento presente; Terapia Dialéctico Conductual (DBT); la cual es un modelo de intervención psicológica basado en la aceptación incondicional del sujeto y la promoción de cambios por medio de una interacción dialéctica entre ambas tendencias. En la Psicoterapia Analítica Funcional, se aplica el análisis de conducta a los intercambios verbales que se producen entre el paciente y el terapeuta en la sesión clínica; y finalmente la Terapia de Activación, la cual actúa bajo la premisa de indicar reforzadores y castigos para guiar el comportamiento individual hacia patrones más normalizados (Hayes, 2004).

Dentro de estas técnicas se ha evidenciado mayor eficiencia de ACT para la población en niños y adolescentes, por tanto, es la que se tomó como relevante dentro del estudio, aunque no se dejan de lado algunos estudios con otras técnicas en esta población. Ya específicamente, dentro de la intervención con niños y adolescentes es importante tener en cuenta que resulta fundamental trabajar con los padres, a los cuales se les realiza una sensibilización para que se hagan partícipes del proceso.

Por ejemplo, en el caso de la ACT dirigida a población infantil, priman los objetivos del análisis funcional de la conducta problema, patrones de conducta que favorecen la evitación de contenidos dolorosos, reforzadores a corto plazo e identificación de direcciones contextuales valiosas en la vida del niño (colegio, amistad o familia). Con la población infantil se usan menos componentes para contactar con el momento presente y el yo como contexto, ya que hay niños que están en un nivel de desarrollo que no les permite relacionar conceptos abstractos, por eso las estrategias terapéuticas son adaptadas dependiendo de la edad y la problemática. (Ferro, 2009).

Se hace necesario visibilizar indicadores bibliométricos que den cuenta del tipo de investigaciones realizadas hasta el momento, teniendo en cuenta autores, tendencias, producciones por año (entre otros) que permitan evaluar el avance en esta área, así como también los cambios en el interés investigativo que puedan ir surgiendo.

Con este análisis se busca mostrar un panorama general acerca del tema y actualizarse en las "novedades" que presentan las terapias de tercera generación en la población infantil y adolescente. Este tipo de análisis bibliométrico es un eje central del ejercicio del psicólogo dentro del campo investigativo y además es una herramienta que permite conocer la producción científica y su impacto a nivel social (Rueda-Clausen, Villa-Roel \& RuedaClausen, 2005). Cabe resaltar que hasta la fecha no hay en Latinoamérica investigaciones que recopilen la temática en mención. Teniendo en cuenta lo anterior, el objetivo de esta investigación fue realizar un análisis bibliométrico de las publicaciones en intervenciones basadas en Terapias de Tercera Generación para niños y adolescentes en Iberoamérica durante los años 2011- 2016.

\section{Método}

\section{Diseño}

El estudio bibliométrico es una herramienta metodológica que parte de la necesidad de cuantificar ciertos aspectos de la ciencia (López, 1996). Parte de la cienciometría que aplica métodos matemáticos y estadísticos a toda la literatura de carácter 
científico, con el objetivo de estudiar y analizar la actividad científica, logrando cuantificar aspectos sociales de la ciencia que según Montero (2007), facilitan un estudio descriptivo mediante un análisis de documentos. Este tipo de metodología proporciona bloques de construcción empírica de la teórica (Schinka \& Velicer, 2003).

\section{Unidad de Análisis}

Para el desarrollo de esta investigación se analizaron 60 artículos científicos publicados entre los años 2011 y 2016 de Iberoamérica y Europa (Alemania, Australia, Canadá, Costa Rica, España, Estados unidos, Suecia, Reino Unido, Países Bajos y Francia) sobre la aplicación clínica de las terapias de tercera generación (ACT, Mindfulness, DBT, terapia de desactivación Modal y Psicoterapia analítico funcional) en niños y adolescentes.

\section{Procedimiento}

La búsqueda de los artículos se llevó a cabo en las bases de datos: PsyNet, Dialnet, Ebsco Host, Elsevier, Proquest, Redalyc, Sage, ScienceDirect y Springer Science; con el propósito de registrar la información más importante de los artículos. Se codificaron los estudios con los siguientes parámetros: (1) año de publicación, (2) nombre de la revista consultada, (3) autores, (4) base de datos, (5) metodología, (6) país de origen, (7) tipo de terapia, (8) población, (9) diagnostico, (10) tratamiento farmacológico, (11) efecto, (12) tamaño del efecto, (13) seguimiento, (14) evaluación pre tratamiento, (15) evaluación post tratamiento, (16) instrumentos y (17) instrumentos alternos. En la segunda fase se recolectó la información de cada uno de los artículos en la base de datos. Finalmente, en la tercera fase se llevó a cabo el análisis de la información teniendo en cuenta las variables que fueron determinadas.

\section{Resultados}

A continuación, se presentan los resultados del análisis de 60 artículos, en los que se informa de la aplicación de las terapias de tercera generación en niños y adolescentes, los cuales se encuentran condensados en los artículos revisados. Estos fueron halladas a través de la búsqueda en las siguientes bases de datos PsyNet, Dialnet, Ebsco Host, Elsevier, Proquest, Redalyc, Sage, ScienceDirect y Springer Science entre otras, de los años 2011 al 2016.

A continuación, se presentan los análisis descriptivos.

Tabla 1.

Año de publicación de los artículos.

\begin{tabular}{ccc}
\hline Año & Frecuencia & Porcentaje \\
\hline 2010 & 1 & 2 \\
\hline 2011 & 11 & 18 \\
\hline 2012 & 14 & 23 \\
\hline 2013 & 9 & 15 \\
\hline 2014 & 16 & 27 \\
\hline 2015 & 6 & 10 \\
\hline 2016 & 3 & 5 \\
\hline Total & 60 & 100 \\
\hline
\end{tabular}

Fuente: Elaboración Propia

En total para el presente análisis se recopilaron 60 artículos (Tabla 1) siendo el de mayor frecuencia de artículos publicados por año, los años 2014 con $27 \%$ y 2012 con $23 \%$.

En la Tabla 2 se registran las revistas en las que se publicaron los resultados de la investigación aplicada en las terapias de tercera generación en niños y adolescentes. Se observa que la revista con mayor frecuencia de artículos publicados en el tema es la International Journal of Behavioral Consultation \& Therapy con un porcentaje del $18 \%$, seguida de la revista Child and Adolescent Mental Health con un porcentaje del $8 \%$.

Tabla 2.

Nombre de las Revistas consultadas.

\begin{tabular}{lcc}
\hline \multicolumn{1}{c}{ Revista } & Frecuencia & Porcentaje \\
\hline $\begin{array}{l}\text { Child and Adolescent } \\
\text { Mental Health }\end{array}$ & 5 & 8 \\
\hline $\begin{array}{l}\text { Cognitive and Behavioral } \\
\text { Practice }\end{array}$ & 2 & 3 \\
\hline
\end{tabular}




\begin{tabular}{lcc}
\hline \multicolumn{1}{c}{ Revista } & Frecuencia & Porcentaje \\
\hline $\begin{array}{l}\text { International Journal of } \\
\text { Behavioral Consultation \& } \\
\text { Therapy }\end{array}$ & 11 & 18 \\
\hline $\begin{array}{l}\text { Journal of Child and } \\
\text { Family Studies }\end{array}$ & 4 & 7 \\
\hline Mindfulness & 3 & 5 \\
\hline Total & 60 & 100 \\
\hline
\end{tabular}

Fuente: Elaboración propia

En la Tabla 3 se presentan los principales autores que publicaron con más frecuencia en el tema de las terapias de tercera generación en niños y adolescentes, teniendo como autor principal a Swart, J con un $7 \%$, seguido de Hayes, S. C. con un $5 \%$.

Tabla 3.

Autores principales con mayor número de artículos dentro de la muestra.

\begin{tabular}{lcc}
\hline \multicolumn{1}{c}{ Autores } & Frecuencia & Porcentaje \\
\hline Hayes, L & 2 & 3 \\
\hline Hayes, S & 3 & 5 \\
\hline Murrell & 2 & 3 \\
\hline Swart, J & 4 & 7 \\
\hline Total & 60 & 100 \\
\hline
\end{tabular}

Fuente: Elaboración propia

En la tabla 4 se presenta el resultado de artículos relacionados con las bases de datos, de las cuales 30 artículos corresponden a Ebsco Host representando un $50 \%$, seguida de la base de datos SpringerScience con un $17 \%$.

Tabla 4.

Bases de datos consultadas.

\begin{tabular}{lcc}
\hline \multicolumn{1}{c}{ Base de datos } & Frecuencia & Porcentaje \\
\hline Ebsco Host & 30 & 50 \\
\hline SpringerScience & 10 & 17 \\
\hline ScienceDirect & 8 & 13 \\
\hline PsyNet & 4 & 7 \\
\hline Elsevier & 4 & 7 \\
\hline Dialnet & 1 & 2 \\
\hline ProQuest & 1 & 2 \\
\hline Redalyc & 1 & 2 \\
\hline
\end{tabular}

\begin{tabular}{lll}
\hline \multicolumn{1}{c}{ Base de datos } & Frecuencia & Porcentaje \\
\hline Sage & 1 & 2 \\
\hline Total & 60 & 100 \\
\hline
\end{tabular}

Fuente: Elaboración Propia

En la tabla 5 se encuentra que la metodología más usada en los artículos revisados sobre la temática, fue la revisión teórica con un $27 \%$, seguido del estudio de naturaleza cuantitativa y con diseño Cuasi-experimental con un $23 \%$ y de diseño de Meta-análisis con un $13 \%$.

Tabla 5.

Metodología usada en los artículos consultados.

\begin{tabular}{lcc}
\hline \multicolumn{1}{c}{ Metodología } & Frecuencia & Porcentaje \\
\hline Revisión Teórica & 16 & 27 \\
\hline Bibliografía & 1 & 2 \\
\hline Meta-análisis & 8 & 13 \\
\hline Bibliografía Meta-análisis & 1 & 2 \\
\hline $\begin{array}{l}\text { Cuantitativo Descriptivo } \\
\text { Correlacional }\end{array}$ & 7 & 12 \\
\hline $\begin{array}{l}\text { Cuantitativo } \\
\text { Exploratorio }\end{array}$ & 4 & 7 \\
\hline $\begin{array}{l}\text { Cuantitativo Cuasi- } \\
\text { experimental }\end{array}$ & 14 & 23 \\
\hline Estudios de Caso & 6 & 10 \\
\hline Análisis de contenido & 2 & 3 \\
\hline Ensayo Clínico & 1 & 2 \\
\hline Total & 60 & 100 \\
\hline
\end{tabular}

Fuente: Elaboración Propia

En la tabla 6 se encuentra que el país con mayores publicaciones en terapias de tercera generación en niños y adolescentes fue Estados Unidos con 35 artículos que equivalen a un $58 \%$, seguido de Australia con un total de 6 artículos lo que equivale al $10 \%$.

Tabla 6.

Países de publicación de los artículos.

\begin{tabular}{lcc}
\hline \multicolumn{1}{c}{ País } & Frecuencia & Porcentaje \\
\hline Alemania & 2 & 3 \\
\hline Australia & 6 & 10 \\
\hline Canadá & 5 & 8 \\
\hline Costa Rica & 1 & 2 \\
\hline
\end{tabular}




\begin{tabular}{lcc}
\hline \multicolumn{1}{c}{ País } & Frecuencia & Porcentaje \\
\hline España & 4 & 7 \\
\hline Estados Unidos & 35 & 58 \\
\hline Inglaterra & 1 & 2 \\
\hline Suecia & 1 & 2 \\
\hline Reino Unido & 1 & 2 \\
\hline Países bajos & 1 & 2 \\
\hline Francia & 1 & 2 \\
\hline Colombia & 1 & 2 \\
\hline Irán & 1 & 2 \\
\hline Total & 60 & 100 \\
\hline
\end{tabular}

Fuente: Elaboración Propia

En la tabla 7, se indica que la terapia con mayor número de artículos revisados es Mindfulness con 20 artículos que equivalen a un 33\%, ACT con 18 artículos correspondientes al $30 \%, 12$ artículos de DBT equivalente al $20 \%$.

Tabla 7.

Tipo de terapia utilizada en los artículos revisados.

\begin{tabular}{lcc}
\hline \multicolumn{1}{c}{$\begin{array}{c}\text { Tipo de Terapia } \\
\text { Psicológica }\end{array}$} & Frecuencia & Porcentaje \\
\hline $\begin{array}{l}\text { Terapia de Aceptación y } \\
\text { Compromiso }\end{array}$ & 18 & 30 \\
\hline $\begin{array}{l}\text { Terapia Cognitivo } \\
\text { Conductual }\end{array}$ & 2 & 3 \\
\hline Mindfulness & 20 & 33 \\
\hline $\begin{array}{l}\text { Terapia Basada en la } \\
\text { Atención Plena }\end{array}$ & 1 & 2 \\
\hline $\begin{array}{l}\text { Terapia de Tercera } \\
\text { Generación }\end{array}$ & 2 & 3 \\
\hline $\begin{array}{l}\text { Terapia Dialectico } \\
\text { Conductual }\end{array}$ & 12 & 20 \\
\hline $\begin{array}{l}\text { Terapia de } \\
\text { Desactivación Modal }\end{array}$ & 4 & 7 \\
\hline $\begin{array}{l}\text { Psicoterapia Analítica } \\
\text { Funcional }\end{array}$ & 1 & 2 \\
\hline Total & 60 & 100 \\
\hline
\end{tabular}

Fuente: Elaboración Propia

De los 60 artículos revisados, 17 de ellos no mencionan las edades de los participantes por lo cual no se tienen en cuenta para el análisis de esta tabla. En la tabla 8 el rango de edad con índice más alto de publicaciones fue el de 10,1 a 15 años con un porcentaje del $49 \%$, seguido de 15,1 a 18 años, lo cual corresponde al $42 \%$.

Tabla 8.

Tamaño de la población.

\begin{tabular}{lcc}
\hline Población en años & Frecuencia & Porcentaje \\
\hline $3-10$ & 1 & 2 \\
\hline $10,1-15$ & 21 & 49 \\
\hline $15,1-18$ & 18 & 42 \\
\hline $18,1-22$ & 3 & 7 \\
\hline Total & 43 & $100 \%$ \\
\hline
\end{tabular}

Fuente: Elaboración Propia

En 11 de los artículos analizados no se menciona el diagnóstico, por lo cual no son tenidos en cuenta en el análisis de la tabla 9, dentro de la cual se observa que en el $14 \%$ estuvieron dirigidos a personas con trastorno límite de personalidad y trastorno de evitación experiencia, en tanto que el $12 \%$ estuvo dirigido a niños con dificultades en atención e hiperactividad.

Tabla 9.

Diagnósticos encontrados en los artículos analizados.

\begin{tabular}{lcc}
\hline Diagnostico principal & Frecuencia & Porcentaje \\
\hline Ansiedad & 2 & 4 \\
\hline Impulsividad & 1 & 2 \\
\hline Depresión & 2 & 4 \\
\hline $\begin{array}{l}\text { Desorden psiquiátrico/ } \\
\text { Trastorno somático }\end{array}$ & 4 & 8 \\
\hline $\begin{array}{l}\text { Familias con problemas } \\
\text { de maltrato y/o } \\
\text { negligencia infantil }\end{array}$ & 1 & 2 \\
\hline $\begin{array}{l}\text { Trastorno de ansiedad } \\
\text { y Trastorno obsesivo } \\
\text { compulsivo }\end{array}$ & 1 & 2 \\
\hline $\begin{array}{l}\text { Dolor crónico, trastorno } \\
\text { obsesivo-compulsivo, y } \\
\text { trastornos de ansiedad }\end{array}$ & 2 & 4 \\
\hline $\begin{array}{l}\text { Comportamientos } \\
\text { agresivos }\end{array}$ & 3 & 6 \\
\hline $\begin{array}{l}\text { Dificultad en atención e } \\
\text { hiperactividad }\end{array}$ & 6 & 12 \\
\hline \begin{tabular}{l} 
Abuso sexual \\
\hline
\end{tabular} & 1 & 2 \\
\hline
\end{tabular}




\begin{tabular}{lcc}
\hline \multicolumn{1}{c}{ Diagnostico principal } & Frecuencia & Porcentaje \\
\hline Ideación suicida & 3 & 6 \\
\hline $\begin{array}{l}\text { Trastorno límite de } \\
\text { personalidad }\end{array}$ & 7 & 14 \\
\hline $\begin{array}{l}\text { Trastorno oposicionista } \\
\text { desafiante }\end{array}$ & 2 & 4 \\
\hline Conducta antisocial & 2 & 4 \\
\hline $\begin{array}{l}\text { Delitos sexuales } \\
\text { Ira }\end{array}$ & 1 & 2 \\
\hline $\begin{array}{l}\text { Abuso y dependencia de } \\
\text { sustancias }\end{array}$ & 1 & 6 \\
\hline $\begin{array}{l}\text { Trastorno de evitación } \\
\text { experiencial }\end{array}$ & 7 & 14 \\
\hline \begin{tabular}{l} 
Total \\
\hline
\end{tabular} & 49 & $100 \%$ \\
\hline
\end{tabular}

Fuente: Elaboración Propia

En la tabla 10 , se muestra que el $92 \%$ de los artículos analizados no señalaban un tratamiento farmacológico, los cuales equivalen a 55 artículos, el $8 \%$ restante, es decir 5 artículos si tenían un tratamiento farmacológico en el estudio de los casos, los cuales estaban guiados para manejo de síntomas ansiosos y depresivos.

Tabla 10.

Tratamiento farmacológico.

\begin{tabular}{ccc}
\cline { 2 - 3 } & Frecuencia & Porcentaje \\
\hline Si & 5 & 8 \\
\hline No & 55 & 92 \\
\hline Total & 60 & 100 \\
\hline
\end{tabular}

Elaboración propia

En la tabla 11, se muestra que en el $88 \%$ de los artículos revisados, es decir 53 de ellos reportaron efectos en el tratamiento principal con el uso de estas terapias de tercera generación. El10 $\%(n=6)$ reportan que existe un incremento en las conductas adaptativas y el $2 \%$ de los artículos sugirió que no existía efecto en el tratamiento principal.

Tabla 11.

Efecto de las terapias de tercera generación.

\begin{tabular}{lcc}
\cline { 2 - 3 } & Frecuencia & Porcentaje \\
\hline $\begin{array}{l}\text { Efecto en el tratamiento } \\
\text { principal }\end{array}$ & 53 & 88 \\
\hline $\begin{array}{l}\text { No hubo efecto en el } \\
\text { tratamiento principal }\end{array}$ & 1 & 2 \\
\hline $\begin{array}{l}\text { Hay incremento en las } \\
\text { conductas adaptativas }\end{array}$ & 6 & 10 \\
\hline Total & 60 & 100 \\
\hline
\end{tabular}

Fuente: Elaboración Propia

En la tabla 12 se reporta que en el $70 \%$ de los artículos revisados los autores no reportan el tamaño del efecto, en el $5 \%$ de los casos indican que el tamaño del efecto es grande $(0.8)$ y el $15 \%$ de los artículos presentan un e tamaño del efecto medio (0.2- 0.79).

Tabla 12.

Tamaño del efecto.

\begin{tabular}{lcc}
\cline { 2 - 3 } & Frecuencia & Porcentaje \\
\hline No reportan & 42 & 70 \\
\hline Menor a 0.2 & 6 & 10 \\
\hline Entre 0.2 y 0.79 & 9 & 15 \\
\hline Mayor a 0.8 & 3 & 5 \\
\hline Total & 60 & 100 \\
\hline
\end{tabular}

Fuente: Elaboración Propia

En la tabla 13, se muestra que en el $55 \%$ de los artículos no realizaron una evaluación pre-tratamiento, el $45 \%$ restante si realizaron la evaluación antes de aplicar la terapia de tercera generación.

Tabla 13.

Evaluación pre tratamiento.

\begin{tabular}{ccc}
\cline { 2 - 3 } & Frecuencia & Porcentaje \\
\hline $\mathrm{Si}$ & 27 & 45 \\
\hline No & 33 & 55 \\
\hline Total & 60 & 100 \\
\hline
\end{tabular}

Fuente: Elaboración Propia 
En la tabla 14, se muestra que el $50 \%$ de los casos que hacen referencia a 25 artículos realizaron la evaluación del post-tratamiento y el $50 \%$ restante no realizaron evaluación luego de la aplicación de la terapia de tercera generación.

Tabla 14.

Evaluación post tratamiento.

\begin{tabular}{ccc}
\cline { 2 - 3 } & Frecuencia & Porcentaje \\
\hline $\mathrm{Si}$ & 28 & 47 \\
\hline No & 32 & 53 \\
\hline Total & 60 & 100 \\
\hline
\end{tabular}

Fuente: Elaboración Propia

En la tabla 15 , se evidencia que en el $47 \%$ de los artículos analizados no se aplicaron los instrumentos; el $10 \%$ de instrumentos se utilizaron para medir sintomatología depresiva, en tanto que el $12 \%$ utilizaron instrumentos de observación del comportamiento infantil. En cuanto a los instrumentos para medir ansiedad, este fue usado en el $7 \%$ de los casos, mientras que los instrumentos para medir afecto se utilizaron en un $5 \%$ de los estudios.

Tabla 15.

Instrumentos.

\begin{tabular}{lcc}
\hline & Frecuencia & Porcentaje \\
\hline Instrumentos de ansiedad & 4 & 7 \\
\hline $\begin{array}{l}\text { Instrumentos de depresión } \\
\text { Instrumentos de aceptación }\end{array}$ & 6 & 10 \\
acción & 2 & 3 \\
\hline $\begin{array}{l}\text { Instrumento de } \\
\text { comportamiento infantil }\end{array}$ & 7 & 12 \\
\hline $\begin{array}{l}\text { Instrumento de habilidades } \\
\text { de Mindfulness }\end{array}$ & 6 & 10 \\
\hline $\begin{array}{l}\text { Instrumento para evaluar } \\
\text { creencias y procesos } \\
\text { cognitivos }\end{array}$ & 1 & 2 \\
\hline $\begin{array}{l}\text { Instrumento para medir } \\
\text { afecto }\end{array}$ & 3 & 5 \\
\hline $\begin{array}{l}\text { Registro de repertorio } \\
\text { relacional }\end{array}$ & 1 & 2 \\
\hline $\begin{array}{l}\text { Instrumentos de depresión, } \\
\text { Escala de resiliencia, Escala } \\
\text { de autoestima }\end{array}$ & 1 & \\
\hline
\end{tabular}

\begin{tabular}{lcc}
\hline & Frecuencia & Porcentaje \\
\hline $\begin{array}{l}\text { Escala de depresión } \\
\text { infantil, Escala de }\end{array}$ & 1 & 2 \\
$\begin{array}{l}\text { sensación de culpa, Escala } \\
\text { de satisfacción con la vida }\end{array}$ & & \\
\hline No reportan & 28 & 47 \\
\hline Total & 60 & 100 \\
\hline
\end{tabular}

Fuente: Elaboración Propia

\section{Discusión}

Las terapias de tercera generación son la versión más actualizada de las terapias del comportamiento, las cuales retoman el análisis de la conducta verbal de B. F. Skinner, incorporando el análisis del lenguaje desde la teoría de los marcos relacionales y asume el contextualismo funcional. Estas terapias de tercera generación se desarrollan a partir del análisis del lenguaje como una mejor aproximación explicativa del comportamiento humano, siendo los eventos privados cómo los sentimientos, emociones y pensamientos, entendidos como conducta verbal.

El objetivo de la investigación fue analizar las características bibliométricas de las publicaciones en terapias de tercera generación en niños y adolescentes, por lo que a continuación se discutirán los hallazgos a la luz de la teoría y aproximaciones futuras a partir de cada uno de los objetivos propuestos.

De los artículos revisados los resultados principales que son coherentes con los objetivos son: se evidencia que desde el 2010 al 2016 se presenta un mayor porcentaje en la publicación de artículos referentes a las terapias de tercera generación en niños y adolescentes. Esto demuestra que naciéndose presenta un mayor interés por la investigación y el estudio de la importancia de las terapias de tercera generación en los últimos años.

En lo referente a la metodología que más se registra en este análisis es la revisión teórica con un $27 \%$ seguido por las investigaciones de diseño cuasi-experimental, aspecto que podría estar relacionado con la necesidad que se ha encontrado para, en ambientes experimentales, evaluar 
la efectividad de las ciencias y las mediciones en las terapias de tercera generación. Por otro lado, dentro de los países con más publicaciones en este ámbito se encuentra Estados Unidos con un número de 35 artículos, esto muestra que la terapia de tercera generación ha adquirido adeptos en otros países diferentes a los de su origen, generando investigaciones que promueven el uso de este nuevo tipo de terapias.

Es necesario plantear que los tipos de terapia de tercera generación con mayores investigaciones encontradas son Mindfulness y la terapia de aceptación y compromiso, encontrándose en mayor medida intervención en estas terapias hacia problemáticas cómo el trastorno límite de personalidad, TDAH y diversos diagnósticos como la ansiedad o inclusive la depresión crónica, situación que podría estar relacionada con hallazgos frente a la efectividad de las mismas en las intervenciones.

Aunque no se han encontrado meta-análisis o análisis bibliométricos dirigidos a evaluar la efectividad de las terapias de tercera generación en problemáticas infanto-juveniles, estos si se han realizado para mostrar la efectividad con grupos poblacionales en general. En los resultados, los autores han planteado que las terapias de tercera generación, especialmente la terapia de ACT, es más efectiva en comparación a los grupos control (Bluett, Homan, Morrison, Levin, \&Twohig, 2014; Powers, ZumVördeSive, Vörding, \& Emmelkamp, 2009). A partir de lo anterior, los resultados de este análisis bibliométrico reflejan hallazgos positivos en la medida de cambio del comportamiento. Sin embargo, debido a los escasos números de estudios de corte experimental y cuasi-experimental publicados en tratamientos de tercera generación, son limitadas las conclusiones que se pueden sacar acerca de la efectividad, siendo necesario aplicar modelos de intervenciones clínicas con mayor control de variables, así como incluir más investigaciones de corte experimental.

Con lo anterior, se podría plantear que la generalización de los datos en las aplicaciones de la Terapia de Aceptación y Compromiso es reducida, dadas las características propias de la investigación de caso único, ya que, desde este tipo de terapia se ha presentado un mayor interés por los aspectos particulares de cada caso, aspecto que en este punto se vuelve una limitación, siendo necesarios también estudios que permitan mayor generalización de los resultados.

Adicional a esto, tal como era planteado por Sanz y García (2015), al predominar el análisis visual de la presentación gráfica de datos dentro de los resultados de los estudios de caso único, se pueden tener limitaciones cómo la tendencia a presentar errores de tipo I, como por ejemplo en el $25 \%$ de estudios de diseños de caso único, se han considerado efectivo un tratamiento que no lo ha sido (Campbell \& Herzinger, 2010 citado por Sanz \& García 2015). De otro lado, se ha encontrado que puede presentarse una baja fiabilidad interjueces cuando se interpretan los resultados partiendo solamente del análisis visual. Es decir, dos observadores pueden mostrar poco acuerdo sobre si un tratamiento es efectivo, así como la relevancia de dicho efecto (Campbell \& Herzinger, 2010 citado por Sanz \& García 2015).

Teniendo en cuenta que el principal objetivo de este análisis fue estudiar y analizar la actividad científica frente a la investigación de terapias de tercera generación en niños y adolescentes, uno de los aspectos centrales en lo que refiere a la efectividad de las mismas, se encuentra un $88 \%$ de efecto en el tratamiento principal en este tipo de terapias, no obstante, solo el $23 \%$ de los artículos mostraron tamaño del efecto, aspecto que podría estar relacionado con la predominancia de la presentación visual de datos como estrategia de análisis de resultados.

Lo anterior lleva a plantear la necesidad de generar mayor tipo de investigaciones de corte experimental, en donde se puedan analizar los resultados de la terapia en comparación con grupos control y se pueda determinar en un mayor grado la eficacia de la aplicación de las terapias de tercera generación en distintos escenarios y que a la vez se pueda analizar dichos efectos a corto, mediano y largo plazo. En lo que respecta al análisis de resultados en los diseños de caso único, ampliamente utilizados desde la Terapia de Aceptación y Compromiso, se pueden 
complementar dicho análisis visual de datos con herramientas de análisis estadístico como los índices para la estimación del tamaño del efecto basado en el no solapamiento de los datos, al proporcionar un método de análisis cuantitativo para evaluar el efecto y de cambio terapéutico. Este análisis estadístico permite evidenciar la magnitud de cambio terapéutico y facilitando la comparación entre distintos estudios, participantes y medidas de instrumentos (Sanz \& García 2015).

De la misma forma, se sugiere para estudios posteriores, tal como también era planteado por Öst (2007), tener en cuenta artículos donde estrictamente se utilicen terapias de tercera generación, que incluyan fase de pre y post tratamiento, en los que se comparen los resultados al inicio y al final de la intervención, evidenciando los cambios y por otro lado, describir protocolos que sean visualmente analizables y donde se reporte tamaños del efecto para que se pueda observar la implicación de las terapias.

En las investigaciones, aunque se prueba la efectividad de las terapias de tercera generación, se continúan usando instrumentos de segunda generación que evalúan constructos que no obedecen a la conceptualización de la inflexibilidad psicológica como eje central de las problemáticas de niños y adolescentes. Por tanto, se propone que el desarrollo de investigaciones posteriores este más beneficiada en el desarrollo de instrumentos psicométricos individualizados, con el fin de ver repertorios de comportamiento y su función en el contexto.

\section{Conclusiones}

Los principales hallazgos de este estudio bibliométrico se condensan en las siguientes conclusiones:

Se muestra un aumento de estudios en cuanto a las terapias de tercera generación en los últimos años, esto se ve justificado con las publicaciones en el 2014, lo que indica que se está generando aceptación e interés en incluir dichas técnicas en el tratamiento psicológico en parte a los resultados parciales frente a su efectividad, pero aun reconociendo la necesidad de ampliar su cuerpo empírico y plantear las investigaciones empleando otro tipo de metodologías adicional a las investigaciones de caso único.

No se puede determinar la magnitud y el efecto del cambio. Así se recomienda que en estudios posteriores sobre las terapias de tercera generación o programas de intervención en niños y adolescentes se tenga en cuenta el establecimiento de una línea de base dado que estudios posteriores pueden estar dirigidos a establecer el poder predictivo sobre el cambio.

Dentro de las investigaciones reportadas se encuentra que existen muy pocas en habla hispana. Esto puede deberse a que dicha terapia es relativamente nueva, lo cual hace que hasta ahora se esté incursionando en la aplicabilidad de la técnica en diferentes estudios con niños y jóvenes en el contexto Iberoamericano. Adicionalmente, son unas terapias que se han desarrollado en el contexto de habla inglesa, debido a su autor principal y hasta diez años después se está actualizando en América e Iberoamérica en los adelantos que existen en estas técnicas y en la Psicología Clínica en general. De hecho, la primera publicación en Iberoamérica fue realizada en el año 1991 por Gómez \& Luciano (1991) denominada "Autocontrol en niños un estudio experimental sobre dos procedimientos en la adquisición de conductas de espera". Siguiendo con lo anterior, se necesitan mayores investigaciones en el contexto Iberamericano, como parte fundamental del proceso de validez de las terapias, ya que existen variables socioculturales relevantes a tener en cuenta, razón por la cual es necesaria su aplicación en diferentes tipos de contextos.

Por otro lado, dentro del análisis realizado se puede evidenciar que el rango de edad presente en la mayoría de los estudios se concentra entre los 10 y 18 años con un 75\% de participación en los estudios. Adicional a esto, la ACT es la más utilizada, sin embargo cabe anotar que existen terapias de tercera generación diseñadas específicamente para población infantil, tales como la Terapia Familiar Integrativa (Greco \& Eifert, 2004 citados por Ferro, 2009) y la Terapia de Interacción Padres e Hijos (Eiberg, 1988 citados por Ferro, 2009), de los cuales no se encontraron registros de estudios en los últimos diez años que permitieran analizar sus hallazgos. 


\section{Referencias}

*Bass, C. V. (2014). A comparison between dialectical behavior therapy, mode deactivation therapy, cognitive behavioral therapy, and acceptance and commitment therapy in the treatment of adolescents. International Journal of Behavioral Consultation and Therapy. 9(2), 4-8. Doi: 10.1037/h0100991

*Becerra, I. G. (2012). Intervención en valores con familias de riesgo social desde la Terapia de Aceptación y Compromiso. Análisis y modificación de conducta, 38, 157-158

*Beckstead, J. (2015) Effectiveness of Acceptance and Commitment Therapy for Depression, Psychological Well-Being and Feeling of Guilt in 7 - 15 years old Diabetic Children. Addictive Behaviors. 49(2), 24-28

*Beckstead, A., Lambert, M., Dubose, C., \& Linehan, M. (2015). Dialectical behavior therapy with American Indian/Alaska Native adolescents diagnosed with substance use disorders: Combining an evidence based treatment with cultural, traditional, and spiritual beliefs. Addictive Behaviors, 51 84-87.

*Bernier, M. T. (2014). Effects and underlying processes of a mindfulness-based intervention with young elite figure skaters: two case studies. Sport Psychologist, 28(3), 302-315.

Bluett, E., Homan, K. J., Morrison, K. L., Levin, M. E., \& Twohig, M. P. (2014). Acceptance and commitment therapy for anxiety and OCD spectrum disorders: An empirical review. Journal of Anxiety Disorder, 28(6), 612- 624.

*Bluth, K. \& Blanton, P. W. (2014). Mindfulness and self-compassion: Exploring pathways to adolescent emotional well-being. Journal of child and family studies, 23(7)1298-1309.

*Ciesla, J. A. (2012). Dispositional mindfulness moderates the effects of stress among adolescents: rumination as a mediator. Journal of Clinical Child \& Adolescent Psychology, 41(6), 760-770.
${ }^{*}$ Coholic, D. A. (2011). Exploring the feasibility and benefits of arts-based mindfulness-based practices with young people in need: Aiming to improve aspects of self-awareness and resilience. Child \& Youth Care Forum, 40(4) 303317.

${ }^{*}$ Crane, R. S. (2012). Competence in teaching mindfulness-based courses: concepts, development and assessment. Mindfulness, 68, 76-84.

Cunha, M., \& Jacinta, M. (2012). Text Anxiety in Adolescents: The role of Self - Criticism and Acceptance and Mindfulness Skills. The Spanish Journal of Psychology ,15(2), 533-543.

Ferro, V. A., Vives, C., Ascanio, L (2009). Novedades en el tratamiento conductual de niños y adolescentes. Clínica y Salud. 20(2), 119-130

*Fine, K. M. (2012). Acceptance-enhanced behavior therapy for trichotillomania in adolescents. Cognitive and Behavioral Practice, 19 (3), 463-471.

*Fix, R. L. (2013). The effects of mindfulness-based treatments for aggression: a critical review. Aggression and Violent Behavior, 18(2) 219-227.

*Fleischhaker, C. B. (2011). Dialectical Behavioral Therapy for Adolescents (DBT-A): a clinical Trial for Patients with suicidal and self-injurious Behavior and Borderline Symptoms with a one-year Follow-up. Child and Adolescent Psychiatry and Mental Health, 5(3). 1-6.

*Geddes, K. D. (2013). Dialectical behaviour therapy for the treatment of emotion dysregulation and trauma symptoms in self-injurious and suicidal adolescent females: A pilot programme within a community-based child and adolescent mental health service. Psychiatry Journal. 20(1) 1-10.

Gómez \& Luciano (1991). Autocontrol en niños: Un estudio experimenta sobre dos procedimientos en la adquisición de conductas de espera. Universidad de Granada. 
Grabovac, A. D. (2011). Mechanisms of mindfulness: A Buddhist psychological model. Mindfulness, 2(3),154-166.

${ }^{*}$ Greco, L., Baer, R., \& Smith, G. (2011). Assessing Mindfulness in Children and Adolescents: Development and Validation of the Child and Adolescent Mindfulness Measure (CAMM). Psychological Assessment, 23(3), 606-614.

Hagen, S. W.-G. (2014). Potential Benefits of Dialectical Behavioral Therapy for Adolescents Receiving Treatment for Borderline Personality Disorder Traits: Implications for Psychiatric Nurses. Nursing and Health, 2(5), 89-99.

*Halliburton, A; Cooper, L (2015) Applications and adaptations of Acceptance and Commitment Therapy (ACT) for adolescents. Journal of Contextual Behavioral Science, 4, 1-11.

*Harnett, P. H. (2012). The contribution of mindfulness-based therapies for children and families and proposed conceptual integration. Child and Adolescent Mental Health, 17(4), 195-208.

*Haydicky, J. S. (2015). Evaluation of MBCT for adolescents with ADHD and their parents: Impact on individual and family functioning. Journal of Child and Family Studies, 24(1), 76-94.

Hayes, S. C. (2004). Acceptance and commitment therapy, relational frame theory, and third wave of behavioral and cognitive therapies. Behavior Therapy, 35, 639-665.

*Hayes, L. B. (2011). Acceptance and commitment therapy for the treatment of adolescent depression: A pilot study in a psychiatric outpatient setting. Mindfulness, 2(2), 86-94.

Hayes, L. B. (2011). Psychological treatment for adolescent depression: Perspectives on the past, present, and future. Behaviour Change, 27(1), 1-18.

Hayes, S. C. (2011). Open, aware, and active: contextual approaches as an emerging trend in the behavioral and cognitive therapies. Annual Review of Clinical Psychology, 7(1) 141-168.

Hayes, S. C. (2012). Acceptance and commitment therapy as a unified model of behavior change. The Counseling Psychologist, 26(1), 976-1002.

Hayes, S. C., Barnes-Holmes, D., \& Wilson, K. G. (2014). Contextual behavioral science: Creating a science more adequate to the challenge of the human condition. Journal of Contextual Behavioral Science, 1(1), 1-16.

*Himelstein, S. H. (2012). A qualitative investigation of the experience of a mindfulness-based intervention with incarcerated adolescents. Child and Adolescent Mental Health, 17(4), 231-237.

*James, A. C. (2011). A preliminary study of an extension of a community dialectic behaviour therapy (DBT) programme to adolescents in the looked after care system. Child and Adolescent Mental Health, 16(1), 9-13.

*Jennings, J. L. (2013). Using mindfulness in the treatment of adolescent sexual abusers: Contributing common factor or a primary modality? International Journal of Behavioral Consultation and Therapy, 8(3-4), 17-22.

*Kallapiran, K; Siew, K; Kirukaran, R; Hancock, K. (2015) Review: Effectiveness of mindfulness in improving mental health symptoms of children and adolescents: a meta-analysis. Child \& Adolescent Mental Health, 20(4), 182-194.

*Khoury, B. L. (2013). Mindfulness-based therapy: A comprehensive meta-analysis. Clinical Psychology Review, 33(6), 763-771.

*Lynk, R; McCay, R; Carter, C; \& Donald, F (2015) Engaging street-involved youth in dialectical behavior therapy: a secondary analysis. Journal of the Canadian Academy of Child and Adolescent Psychiatry, 24(2), 116-122.

López, P. (1996). Introducción a la Bibliometría. Valencia, PromoLibro. 
*Luciano, C. R.-T.-M.-L. (2011). A relational frame analysis of Defusion interactions in Acceptance and Commitment Therapy. A preliminary and quasi-experimental study with at-risk adolescents. International Journal of Psychology and Psychological Therapy, 11(2) 165-182.

Madriz, L. F. (2012). Terapia de aceptación y compromiso: descripción general de una aproximación con énfasis en los valores personales. Revista de ciencias sociales, 138. 101-110.

*Mehlun, M., Rambertg, M., Tormoen, M., Haga, E., Diep, L., Stanly, B. ... \& Groholt, B (2016) Dialectical Behavior Therapy Compared with Enhanced Usual Care for Adolescents With Repeated Suicidal and Self-Harming Behavior: Outcomes Over a One-Year Follow-Up. Journal of the American Academy of Child \& Adolescent Psychiatry, 55(4), 295-300.

*Miller, A. L.-P. (2011). Adapting dialectical behaviour therapy for children: Towards a new research agenda for paediatric suicidal and non-suicidal self-injurious behaviours. Child and adolescent mental health, 16(2), 116-121.

*Milligan, K. B. (2013). Using Integra Mindfulness Martial Arts to Address Self-regulation Challenges in Youth with Learning Disabilities: A Qualitative Exploration. Journal of Child and Family Studies, 24(3), 1-14.

Montero, I. \& León, O. (2002). Clasificación y descripción de las metodologías de investigación en Psicología. Revista Internacional de Psicología Clínica y de la Salud / International Journal of Clinical and Health Psychology, 2 (3), 503-508.

Moreno, C. (2012). Terapias Cognitivo - Conductuales de tercera generación: la atención plena. Revista Internacional de Psicología. Vol. 12 No.1, 1-17.

*Murrell, A. R. (2014). State of the research \& literature address: ACT with children, adolescents and parents. International Journal of Behavioral Consultation and Therapy, 2(4), 531-543.
*Murrell, A. R. (2014). An acceptance and commitment therapy approach to adolescent suicide. International Journal of Behavioral Consultation and Therapy, 9(3), 41-46.

*Newring, K. A. (2012). Functional Analytic Psychotherapy with juveniles who have committed sexual offenses. International Journal of Behavioral Consultation and Therapy, 7(2), 102-110.

Öst, L.-G. (2007). Efficacy of the third of behavioral therapies: A systematic review and metaanalysis. Behaviour Research and Therapy, 72, 296- 321.

Öst, L. G. (2014). The efficacy of Acceptance and Commitment Therapy: An updated systematic review and meta-analysis. Behaviour research and therapy, 61(1), 105-121.

*Perepletchikova, F. \& Goodman, G. (2014). Two approaches to treating preadolescent children with severe emotional and behavioral problems: Dialectical behavior therapy adapted for children and mentalization-based child therapy. Journal of Psychotherapy Integration, 24(4), 298-312.

Petter, M. C. (2013). The role of trait mindfulness in the pain experience of adolescents. The Journal of Pain, 14(2), 1709-1718.

*Powers, M. B., ZumVördeSive, M., Vörding, P., \& Emmelkamp, M. (2009). Acceptance and Commitment Therapy: A Meta-Analytic Review. PsychotherPsychosom, 78(2), 73 - 80.

Ribero-Marulanda, S. \& Agudelo-Colorado, L. (2016). La aplicación de la terapia de aceptación y compromiso en dos casos de evitación experiencial. Avances en Psicología Latinoamericana, 34(1), 29-46. doi: dx.doi. org/10.12804/ apl34.1.2016.03

Rector, N. A. (2013). Acceptance and commitment therapy: Empirical considerations. Behaviortherapy, 44(2), 213-217.

Rueda-Clausen, C., Villa-Roel, C., \& Rueda-Clausen, C. E. (2005). Indicadores bibliométricos: origen, aplicación, contradicción y nuevas propuestas. MedUNAB, 8(1), 29-36. 
*Ruiz, F. J. (2012). Acceptance and commitment therapy versus traditional cognitive behavioral therapy: A systematic review and meta-analysis of current empirical evidence. International journal of psychology and psychological therapy, 12(3), 333-358.

*Ruiz \& Perete (2015) Application of a relational frame theory account of psychological flexibility in young children. Psicothema. 27(2), 114119. doi:10.7334/psicothema2014.195

Sanz \& García (2015). Técnicas para el análisis de diseños los de caso único en la práctica clínica: ejemplos de aplicación en el tratamiento de víctimas de atentados terroristas. Clínica y Salud, 26(3), 167-180.

Schinka, J., Weiner, I., \& Velicer, W. (2003). Handbook of psychology. Research methods in psychology, 3-424.

Smout, M. F. (2012). The empirically supported status of acceptance and commitment therapy: An update. Clinical Psychologist, 16(3), 97-109.

*Swain, J. H. (2013). Acceptance and Commitment Therapy for anxious children and adolescents: study protocol for a randomized controlled trial. Trials, 14(14), 73-78.

*Swart, J. (2014). A comparative study of mode deactivation therapy (MDT) as an effective treatment of adolescents with suicidal and non-suicidal self-injury behaviors. International Journal of Behavioral Consultation and Therapy, 1(2), 130-144.

*Swart, J. (2014). A comparative treatment efficacy study of conventional therapy and mode deactivation therapy (MDT) for adolescents with conduct disorders, mixed personality disorders, and experiences of childhood trauma. International Journal of Behavioral Consultation and Therapy, 9(1), 23-29.

*Swart, J. \&. Apsche, J. (2014). Mindfulness, mode deactivation, and family therapy: A winning combination for treating adolescents with complex trauma and behavioral problems. International Journal of Behavioral Consultation and Therapy, 9(2), 9-14.

*Thoder, V. J. (2011). An independent evaluation of mode deactivation therapy for juvenile offenders. International Journal of Behavioral Consultation and Therapy, 7(1), 41-46.

Thomas G \& Atkinson, C (2016) Measuring the effectiveness of a mindfulness-based intervention for children's attentional functioning. Educational \& Child Psychology, 33(1), 51-64.

*Tormoen, A. J.-L. (2014). Feasibility of Dialectical Behavior Therapy with Suicidal and SelfHarming Adolescents with Multi-Problems: Training, Adherence, and Retention. Archives of Suicide Research, 18(4), 432-444.

Twohig, M., Hayes, S., \& Berlin, K. (2008). Terapia de aceptación y compromiso para niños con problemas externalizantes. En L. Greco, \& S. Hayes. Acceptance \& Mindfulness treatments for children \& adolescents. 163-186.

*Van de Weijer-Bergsma, E. F. (2012). The effectiveness of mindfulness training on behavioral problems and attentional functioning in adolescents with ADHD. Journal of child and family studies, 21(5), 775-787.

*Vickery, C. \& Dorjee, D. (2016) Mindfulness Training in Primary Schools Decreases Negative Affect and Increases Meta-Cognition in Children. Frontiers in Psychology. 6: 2025. doi: 10.3389/fpsyg.2015.02025.

Zenner, C. H.-K. (2014). Mindfulness-based interventions in schools-a systematic review and meta-analysis. Frontiers in Psychology, 5 (2), 603-610.

\footnotetext{
*Estos artículos fueron incluidos en el análisis bibliométrico.
} 